\title{
The Economics of Roadside Bombs ${ }^{*}$
}

\author{
Matthew A. Hanson ${ }^{\dagger}$ \\ College of William and Mary \\ College of William and Mary \\ Department of Economics \\ Working Paper Number 68
}

December 2007

\footnotetext{
* This paper was previously titled “"Are Improvised Explosive Devices a Giffen Good?”

${ }^{\dagger}$ The author is a student in the Department of Economics at the College of William \& Mary, and can be reached at mahans@wm.edu or by mail at CSU 0304, P.O. Box 8793, Williamsburg, VA 23186. The author would like to thank Tom Allen, Tyler Cowen, Steven Davis, Philip deCamp, Martin Feldstein, Matt Gibbons, Keith Green, Jeff Jaworski, Zubin Jelveh, Carl Moody, Dick Polin, Felix Salmon, Martin Schmidt, Thaddeus Templeton, Alanna Whytock, the members of the NBER Economics of National Security Working Group, and numerous colleagues at the Institute for Defense Analyses for their helpful comments, discussions, and support of economic research on counterinsurgency in Iraq. This research was supported by a grant from the Charles Center at the College of William \& Mary. All errors and omissions are the sole responsibility of the author.
} 
COLLEGE OF WILLIAM AND MARY

DEPARTMENT OF ECONOMICS

WORKING PAPER \#68

December 2007

\title{
The Economics of Roadside Bombs
}

\begin{abstract}
The U.S. military has been criticized for its failure to stop the Iraqi insurgency's use of improvised explosive devices (IEDs), which have caused most of the Coalition casualties. We use an instrumental variables approach to estimate the insurgent responses to U.S. military countermeasures. We find that the number of IED attacks (including unobserved attacks) goes up when attacks are made more costly to conduct, suggesting that IED attacks are inferior and may even be a Giffen good. A major benefit of IED countermeasures therefore comes in reducing non-IED attacks. Evaluations of the U.S. military's \$13 billion counter-IED effort have thus significantly understated its success.
\end{abstract}

JEL Codes: C32, D74, H56

Keywords: Iraq War; Instrumental Variables; Substitution Effect; Inferior Goods; Giffen Behavior; Terrorism

Matthew A. Hanson

Department of Economics

College of William and Mary

Williamsburg, VA 23187-8795

mahans@wm.edu 


\section{Introduction}

This paper presents an economic analysis of one major part of the war in Iraq - the battle against improvised explosive devices (IEDs). Economic analysis of warfare has been an active area of research for decades (e.g. Schelling (1960)), and has been applied to insurgency (e.g. Leites and Wolf (1970)), terrorism (e.g. Enders and Sandler (2006), Enders, Sandler and Cauley (1991), Jaeger and Paserman (2006), Rosendorff and Sandler (2004), Sandler and Enders (2002)), and specifically the war in Iraq (e.g. Davis, Murphy and Topel (2006)). We present empirical results of maximizing behavior in the Iraqi insurgency, its relation to consumer and producer theory, and implications for the fight against IEDs.

Since the invasion of Iraq in March 2003, the insurgency that has arisen to fight the Coalition has killed 3,783 and wounded 27,753 U.S. soldiers 11 Of the deaths, 3,100 have been killed due to insurgent action, and of these, 1,617 have died due to IEDs. IEDs, also known as roadside bombs, are a "device placed or fabricated in an improvised manner incorporating destuctive, lethal, noxious, pyrotechnic, or incendiary chemicals and designed to destroy, incapacitate, harass, or distract." $2^{2}$

The United States military has spent over $\$ 13$ billion on the Joint IED Defeat Organization (JIEDDO), an agency unifying the efforts of all the military services to defeat these bombs. JIEDDO and the overall counter-IED effort have been criticized for their apparent inability to stop or even reduce the number of IED attacks. A Boston Globe news article referred to JIEDDO as "the controversial office that has spent billions of dollars but failed to curb the biggest killer of American troops," and noted that "lawmakers have become increasingly frustrated by its secrecy and apparent lack of progress in stemming the roadside bomb threat." 3 However, no analysis has yet estimated the impact of IED countermeasures on other types of attacks, which are causally linked due to resource constraints. Because insurgents do not have infinite resources, if a countermeasure increases the resources required

\footnotetext{
${ }^{1}$ The Coalition is made up of 33 nations, but the United States has contributed the overwhelming majority of the troops and funding from the initial occupation to the present day. Killed and wounded in action numbers are as of September 17, 2007.

${ }^{2}$ Definition from the Joint IED Defeat Organization.

${ }^{3}$ Bender, Bryan, "War strategy critic to review IED office," Boston Globe, April 16, 2007.
} 
for a successful attack, ceteris paribus, it decreases the resources available for non-IED attacks.

In this analysis, we consider the possibility that IED attacks are an inferior or Giffen good. Other suggestions of possible Giffen goods have included insurance (Hoy and Robson 1981), kerosene (Bopp 1983), quinine water for rats (Battalio, Kagel and Kogut 1991), and rice or wheat in China (Jensen and Miller 2007) 4 We can treat the percentage of IED attacks that are effective as an inversely correlated proxy for the price of an IED attack, since a decrease in the percentage of IED attacks that are effective increases the resources necessary to conduct an effective IED attack. Considering the graph presented in Figure 1. IED attacks appear to increase with a decrease in the percentage of IED attacks that are effective - suggesting the possible existence of Giffen behavior. If the insurgents are resource-constrained, the increase in the number of IED attacks attempted comes at the cost of other types of attacks.

If IED attacks are a normal good, evaluations of the counter-IED effort that do not consider its impact on non-IED attacks have overstated the counter-IED effort's success (because they do not consider the additional non-IED attacks that result). If IED attacks are an inferior or Giffen good, however, evaluations of the counter-IED effort that do not consider its impact on non-IED attacks have understated the counter-IED effort's success (because they do not consider the decrease in non-IED attacks that results).

When countermeasures reduce the returns to an attack (and increase the resources required for success), they are likely to change insurgent behavior. Benmelech and Berrebi (2007) found that Palestinian organizations conducting suicide bombings engage in optimizing behavior - bombers with higher levels of human capital are assigned to higher-value targets. Enders and Sandler (1993), studying transnational terrorism, found that metal detectors in airports and the hardening of buildings decreased skyjackings and attacks against embassies but increased hostage-taking and assassinations - close substitutes for

\footnotetext{
${ }^{4}$ We note that the Giffen characteristic is consumer behavior and not a characteristic of the good itself, and that the proper terminology is Giffen behavior rather than denoting the good itself as Giffen. As we only consider a point elasticity for IED attacks, we use the more casual term Giffen good to denote the existence of Giffen behavior in the observed sample.
} 


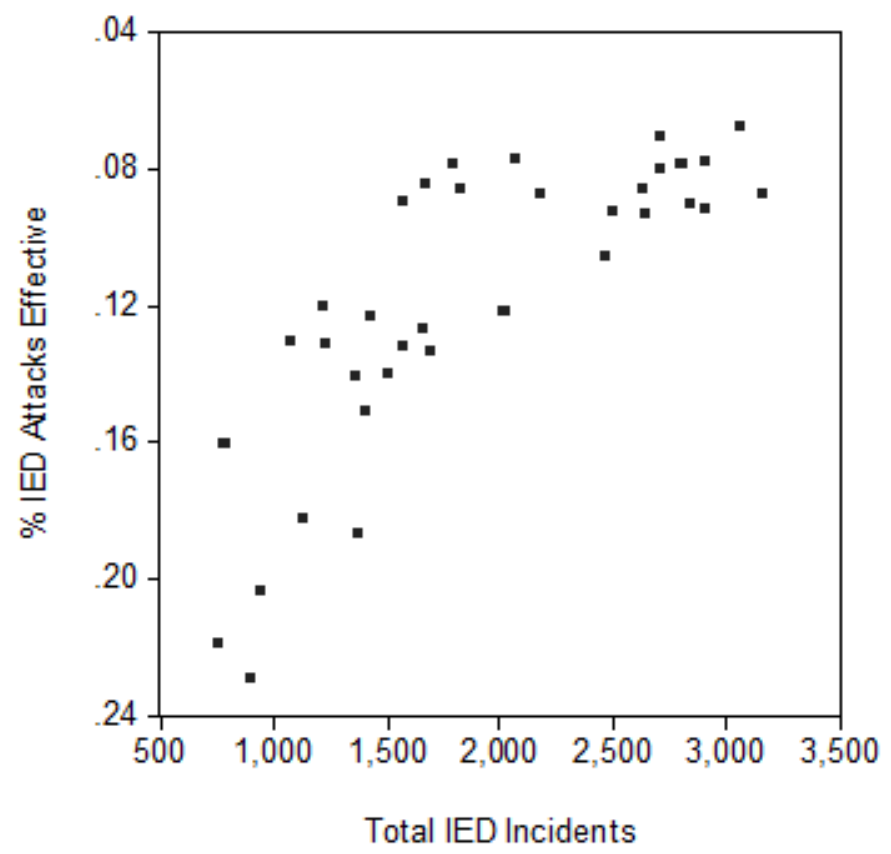

Figure 1: A Proxy for the Demand Curve for IED Attacks.

transnational terrorists. These empirical results suggest that the different attack methods of transnational terrorism are normal goods.

To be a Giffen good, a good must be inferior and absorb a large portion of the consumer's budget. IED attacks make up a large portion of the insurgency's efforts. Because of the high-profile nature of IED attacks, demand for them may be inelastic. These conditions (which are specific to the Iraqi insurgency, as opposed to transnational terrorism) increase the probability that IED attacks are a Giffen good.

We demonstrate below that a Coalition countermeasure which reduces the probability than an IED attack will be effective has no statistically significant impact on the number of observed IED attacks the insurgents conduct. However, these innovations do reduce the number of non-IED attacks, and may actually increase the number of unobserved IED attacks. These facts suggest with strong likelihood that IED attacks may be inferior, or even Giffen. The effects of these countermeasures therefore have been significantly understated. 


\section{Theory, Data, and Methodology}

We consider the case of insurgent groups as consumers purchasing goods (one good being IED attacks, the other being all other goods - non-IED attacks). Adapting the choicetheoretic model of Landes (1978):

$$
E\left[D_{I}\right]=p_{I} D_{I, S}+\left(1-p_{I}\right) D_{I, F}
$$

where $E\left[D_{I}\right]$ is the expected damage inflicted if the insurgents attempt an IED attack, $p_{I}$ is the probability that an IED attack will be successful, $D_{I, S}$ is the damage from a successful IED attack, and $D_{I, F}$ is the damage from an unsuccessful IED attack, with $D_{I, S}>D_{I, F} 5^{5}$ Insurgents conduct an attack if $E\left[D_{I}\right]$ exceeds the cost of an attack.

For a fixed number of attempted IED or non-IED attacks $I, N$, the number of effective attacks is distributed according to $I_{S} \sim \operatorname{Binomial}\left(I, p_{I}\right), I_{N} \sim \operatorname{Binomial}\left(N, p_{N}\right)$. Using this formulation to expand the choice-theoretic model to the aggregate number of IED attacks and non-IED attacks attempted, we can therefore presume that insurgents maximize the damage they inflict on the Coalition subject to resource constraints:

$$
\begin{array}{cc}
\max & E\left[D\left(I_{S}, N_{S}\right)\right] \\
\text { s.t. } & a I+b N \leq R \\
& E\left[I_{S}\right]=p_{I} I, E\left[N_{S}\right]=p_{N} N
\end{array}
$$

where $D$ is a damage function that increases monotonically with the number of successful IED attacks $I_{S}$ and the number of successful non-IED attacks $N_{S}, R$ is the resources available to the insurgents, and $a$ and $b$ represent resources expended for an attempted IED attack or an attempted non-IED attack. With limited assumptions on the behavior of this

\footnotetext{
${ }^{5}$ Successful corresponds to the JIEDDO definitions below of effective IED attacks, while unsuccessful corresponds to ineffective, found and cleared, or unobserved attempts. Here, we use the terminology successful to denote the subset of attacks that are damage-inflicting, and effectiveness to denote the probability that a given attack will be successful. An attack may or may not be damage-inflicting.

${ }^{6}$ This problem can also be formulated as utility maximization, with the condition that utility increases monotonically with damage inflicted.
} 
system $[7$ it is analogous to a consumer maximizing utility by selecting a bundle of goods or a firm maximizing output.

Relabel the expected resources expended per effective IED attack as $f=\frac{a}{p_{I}}$ and per effective non-IED attack as $g=\frac{b}{p_{N}}$, assuming $a$ and $b$ constant. The solution to the maximization problem can be written as

$$
\begin{aligned}
\bar{I} & =\bar{I}(f, g, R) \\
\bar{N} & =\bar{N}(f, g, R) \\
\bar{\lambda} & =\bar{\lambda}(f, g, R)
\end{aligned}
$$

with $\lambda$ as the Lagrange multiplier, interpreted as the marginal damage resulting from additional resources. This formulation represents the choices the insurgents make (selecting $I$ and $N$ rather than $I_{S}$ or $N_{S}$ ). The comparative-static derivatives are 8

$$
\begin{aligned}
\frac{\partial \bar{I}}{\partial R}= & \frac{g D_{I N}-f D_{N N}}{|\bar{H}|} \\
\frac{\partial \bar{N}}{\partial R}= & \frac{f D_{I N}-g D_{I I}}{|\bar{H}|} \\
& \text { and } \\
\frac{\partial \bar{I}}{\partial f}= & -\frac{g^{2} \bar{\lambda}}{|\bar{H}|}-\bar{I} \frac{\partial \bar{I}}{\partial R} \\
\frac{\partial \bar{N}}{\partial f}= & \frac{f g \bar{\lambda}}{|\bar{H}|}-\bar{I} \frac{\partial \bar{N}}{\partial R}
\end{aligned}
$$

We do not directly observe the cost of an IED or non-IED attack, denoted above as $a$ and $b$. However, we do observe the benefits - the probability that an attack will be successful. We therefore can investigate the effect of an exogenous decrease in the benefit of conducting an IED attack - an exogenous decrease in $p_{I}$. According to the choice-theoretic

\footnotetext{
${ }^{7}$ Diminishing marginal returns to IED and non-IED attacks, and small or no complementary effects of damage across IED and non-IED attacks.

${ }^{8}$ We use subscripts to indicate a partial derivative.
} 
model outlined above, a decrease in the benefits has exactly the same effect on the number of IED attacks conducted as some increase in the costs would have. We can therefore treat a decrease in the benefits of attempting IED attacks exactly as we would treat an increase in the cost. This formulation allows estimation of Equations 5 and 6 using data from publicly available U.S. government sources ${ }^{9}$

We therefore consider the goods as attempted IED and non-IED attacks, the mechanism through which they provide benefits to the insurgency as effectiveness, and the price of an attempt as being exactly equivalent to the decrease in benefits that comes from decreased effectiveness.

In the above formulations, the quantities $\bar{I} \frac{\partial \bar{I}}{\partial R}$ and $\bar{I} \frac{\partial \bar{N}}{\partial R}$ are the income effect. If $\frac{\partial \bar{I}}{\partial R}<$ 0 , IED attacks are an inferior good - quantity demanded decreases with an increase in resources. The Giffen case, $\frac{\partial \bar{I}}{\partial f}>0$, occurs only if $\frac{\partial \bar{I}}{\partial R}<0$ and $\left|\bar{I} \frac{\partial \bar{I}}{\partial R}\right|>\left|\frac{g^{2} \bar{\lambda}}{|\bar{H}|}\right|$, that is, the income effect exceeds the substitution effect.

A detailed description of the data collection is presented in Appendix A. A time-series plot of the total number of IED attacks and the number of effective IED attacks is presented in Figure 2,

In order to make these reduced-form equations operational, we use the effectiveness of IED attacks (contemporaneously and with one lag) as a measure of changes in the price (the resources required) to carry out IED attacks. There has been no systematic effort on the scale of JIEDDO to reduce the effectiveness of non-IED attacks, so we therefore assume that this change can be appropriately modeled by a linear trend. As a proxy for the resource constraint, we use the numbers of IED and non-IED attacks in the previous period 10 To allow for differing autoregressive effects, we split these into two variables in each equation.

The effectiveness of IED and non-IED attacks may be endogenous - in particular, it

\footnotetext{
${ }^{9}$ There is no data available on the number of effective non-IED attacks, so the model is incomplete if the goods considered are effective attacks rather than attempted attacks.

${ }^{10}$ Although this proxy may introduce measurement error, the instruments for the effectiveness of IED attacks are likely to be exogenous with respect to the resource constraint, and therefore allow identification of variation due to changes in IED effectiveness rather than in the resource constraint. The results may therefore be less susceptible to the adverse effects of measurement error due to the use of these instrumental variables.
} 


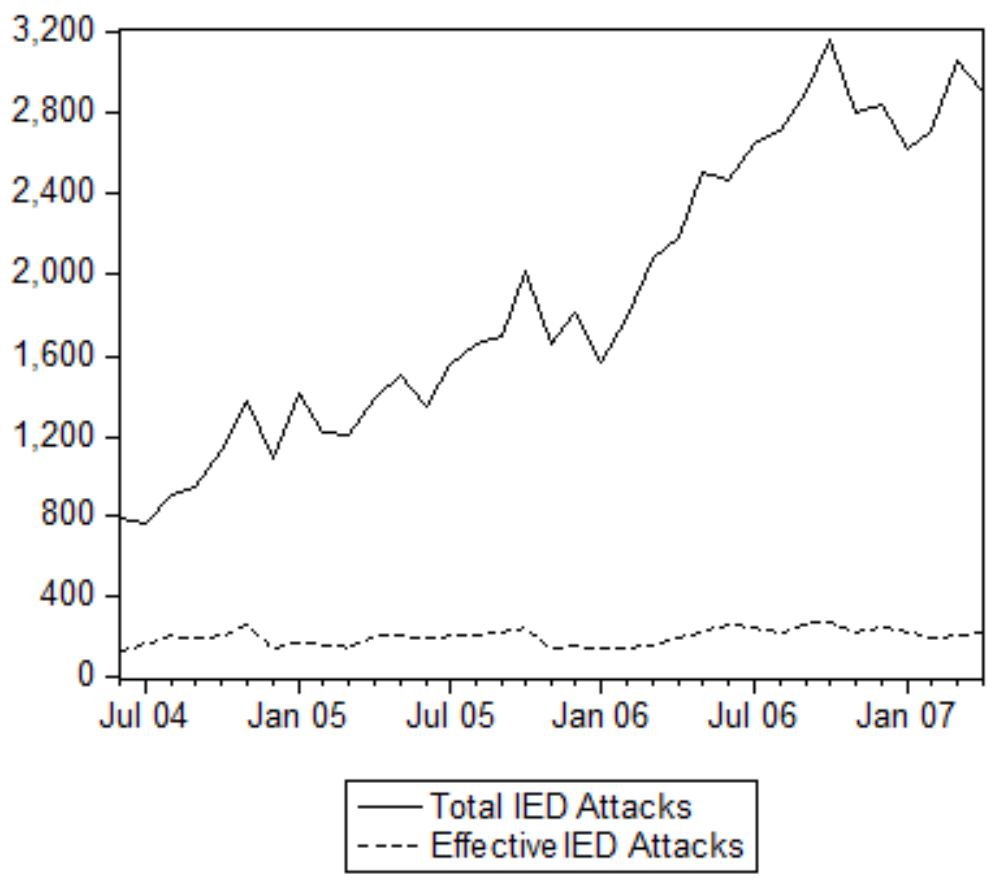

Figure 2: Total IED Incidents and Effective IED Incidents.

may be vary simultaneously with the number of IED and non-IED attacks attempted. Furthermore, there may exist correlations between the effectiveness of IED attacks and non-IED attacks. We therefore require an instrumental variable that is exogenous.

IEDs are triggered with a variety of systems - by radio control, command wire, pressure plate (similar to a landmine), passive infrared sensors, or cell phones. Contemporaneously, the percentage of IEDs that are not triggered by radio control are exogenous once past IED effectiveness (the factor that determines what types of attacks to attempt this period) is controlled for. The percentage of non-radio-control triggers used is determined by Coalition jammer use, which is not a function of contemporaneous insurgent activity.

Jammer use is, perhaps paradoxically, exogenous with respect to IED and non-IED attacks. Coalition forces use all the IED countermeasures available to them since the marginal cost is extremely low, and the available IED countermeasures are the result of spending decisions made in previous periods. The marginal cost of additional use of IED jamming 
systems once they have been fielded is only the electrical power to operate them. Although it is possible that these systems could be deployed in anticipation of higher insurgent effectiveness in the future (making jammer use endogenous), JIEDDO has been criticized for being too slow to react to insurgent innovations. A paper written by three field-grade military officers, Ellis, Rodgers and Cochran (2007), describes JIEDDO as being mired in red tape and unable to effectively respond to new developments in insurgent IED technology 11 Similar issues are raised in Adamson (2007) - authored by the former operations officer of JIEDDO. While JIEDDO has been able to identify some trends in the counter-IED fight, it is virtually impossible to predict insurgent activity, and the long lag in acquisition decisions to deployment of systems prevents these systems from being endogenous responses to contemporaneous insurgent activity. We therefore assume that jammer use is exogenous.

We also assume that insurgents cannot detect changes in countermeasures activity, formulate a response, and execute a response in the same period. Therefore, the decisions about what trigger use to attempt are not a function of contemporaneous countermeasures use. However, the triggers actually observed are a function of countermeasures use, which is exogenous. The use of jammers prevents radio frequency emissions that could trigger an IED. When a jammer is successful, those attempted attacks are not observed because the IED does not detonate. This effect necessarily reduces the observed radio control detonated IEDs and causes more non-radio control triggers to be in the population of observed IEDs. Therefore, use of jammers results in a contemporaneous reduction in the percentage of IEDs observed using radio control triggers. We can use this association between non-radio control trigger use and IED effectiveness to represent the decrease in IED effectiveness due to jammer use.

In order to control for innovations in insurgent technology (which may have both direct and spillover effects), we use passive infrared trigger use as a proxy. These triggers are generally used only with explosively formed penetrator IEDs, which, although they are highly lethal, require a high degree of technical sophistication to construct and emplace.

\footnotetext{
${ }^{11}$ This paper is at http://www.jfsc.ndu.edu/current_students/documents_policies/documents/jca_cca_awsp/.
} 
We therefore estimate:

$$
\begin{aligned}
\widehat{\Delta I}_{t} & =h_{1}\left(\widehat{\Delta E}_{t}, \Delta E_{t-1}, \Delta I_{t-1}, \Delta N_{t-1}\right) \\
\widehat{\Delta N}_{t} & =h_{2}\left(\widehat{\Delta E}_{t}, \Delta E_{t-1}, \Delta I_{t-1}, \Delta N_{t-1}\right) \\
\widehat{\Delta E}_{t} & =h_{3}\left(\Delta G_{C W, t}, \Delta G_{P P, t}, \Delta G_{P I, t}, \Delta G_{C P, t}\right)
\end{aligned}
$$

where $I$ is the number of IED incidents, $N$ is the number of non-IED attacks, $E$ is the average effectiveness of an IED attack, $G_{C W}$ is observed command wire triggers, $G_{P P}$ is observed pressure plate triggers, $G_{P I}$ is observed passive infrared triggers, and $G_{C P}$ is observed cell phone triggers. $\widehat{\Delta E}_{t}$ and $\Delta E_{t-1}$ are proxies for $f$, while $\Delta I_{t-1}$ and $\Delta N_{t-1}$ are proxies for $R$ in Equations 5 and 6 .

The equation error terms are almost certainly correlated: an insurgent group choosing to conduct one more IED attack necessarily cannot conduct as many non-IED attacks, and vice versa. We therefore use system estimation methods to increase efficiency. We conducted estimation using both the three-stage least squares (3SLS) and generalized method of moments (GMM) estimators. The two estimates produced very similar results for parameter estimates. We present the GMM estimates here due to their higher degree of statistical significance; all hypothesis tests described below are the same in the 3SLS model provided that the $10 \%$ confidence level is accepted. The 3SLS estimation results are presented in Appendix B.

\section{Empirical Results}

We find that all of the series are unit root processes ${ }^{12}$ Therefore, we estimate the relationships in first differences ${ }^{13}$ All variables except the instruments (which take on zero values) are expressed in logarithms.

The specification is robust to the removal of some variables (for example, the effective-

\footnotetext{
${ }^{12}$ Testing was done with the augmented Dickey-Fuller test and the efficient unit root test outlined in Elliot, Rothenberg and Stock (1996). Both showed a strong probability of the presence of a unit root.

${ }^{13}$ There is little evidence that any cointegrating relationships exist in the data.
} 
ness variables in the IED equation), but they vary wildly when the constants are excluded. As the constants are one of the proxies used for total insurgent resources (thereby representing a linear trend in levels), this effect is likely due to omitted variable bias. The equations were also estimated with linear time trends (quadratic trends in levels); these trends were not individually or jointly significant and were thus omitted.

The orthogonality conditions are:

$$
\begin{aligned}
\mathbb{E}\left[\left(\Delta I_{t}-h_{1}\left(\widehat{\Delta E}_{t}, \Delta E_{t-1}, \Delta I_{t-1}, \Delta N_{t-1}\right)\right) \mathbf{X}\right] & =\mathbf{0} \\
\mathbb{E}\left[\left(\Delta N_{t}-h_{2}\left(\widehat{\Delta E}_{t}, \Delta E_{t-1}, \Delta I_{t-1}, \Delta N_{t-1}\right)\right) \mathbf{X}\right] & =\mathbf{0}
\end{aligned}
$$

where $\mathbf{X}$ is an $8 \times 1$ vector of a constant, the four instruments, $\Delta E_{t-1}, \Delta I_{t-1}$, and $\Delta N_{t-1}$.

Estimation using a HAC-consistent weighting matrix produces:

$$
\begin{aligned}
& \widehat{\Delta I_{t}}=\underset{(0.016)}{0.064^{* *}}-\underset{(0.204)}{0.004} \widehat{\Delta E_{t}}+\underset{(0.156)}{0.077} \Delta E_{t-1}-\underset{(0.145)}{0.315^{* *}} \Delta I_{t-1}-\underset{(0.048)}{0.074} \Delta N_{t-1} \\
& \left(T=34 \quad \bar{R}^{2}=0.095 \quad \hat{\sigma}=0.116\right) \\
& \widehat{\Delta N}_{t}=\underset{(0.038)}{0.132^{* *}}+\underset{(0.326)}{1.373^{* *}} \widehat{\Delta E}_{t}+\underset{(0.419)}{1.011^{* *}} \Delta E_{t-1}-\underset{(0.479)}{0.890^{*}} \Delta I_{t-1}-\underset{(0.196)}{0.239} \Delta N_{t-1} \\
& \left(T=34 \quad \bar{R}^{2}=0.221 \quad \hat{\sigma}=0.338\right) \\
& \text { (standard errors in parentheses) } \\
& \text { ** significant at the } 5 \% \text { confidence level }
\end{aligned}
$$

In order to test for overidentifying restrictions, we use the methodology in Hansen (1982). Hansen shows that the minimized value of the objective function times the number of observations, $N_{T}$, is distributed according to $\chi^{2}(r-l)$, where $r-l$ is the number of restrictions imposed beyond the just-identified case. We find $N_{T}=7.508$ with $r-l=6$, and accept the null hypothesis of no overidentifying restrictions. 


\section{Discussion}

\subsection{Are IED Attacks an Inferior or Giffen Good?}

We find that the coefficient on effectiveness in the IED equation is close to zero, suggesting that demand for IED attacks is inelastic. Furthermore, because a decrease in $\alpha \Delta I+\beta \Delta N$ will have a more negative effect on non-IED attacks than on IED attacks (for $\alpha>0, \beta>$ $0)$, IED attacks are an inferior good (since they increase proportionally with a decline in resources). We find a statistically insignificant positive effect of IED countermeasures on attempted IED attacks. However, it is important to note that the estimated effect is actually a lower bound on the effect of IED countermeasures on IED attacks. The estimated effect is actually higher due to the fact that not all IED attacks are observed.

We observe a subset of the total number of IED attacks. Insurgents may attempt an IED attack, but if the trigger fails (quite possible because the devices are, by definition, improvised), the Coalition may not observe it. However, this type of attack uses the same resources as any other ineffective attack, and insurgents do not know in advance which attacks will be ineffective.

When jammers are deployed, they can have one of two contemporaneous effects. Either the jammers are completely ineffective (in which case no change is observed), or the jammers have some effect. Because the instruments have some contemporaneous correlation with $\Delta I_{t}$, there is evidence that jammers are not completely ineffective. Two effects occur from jammer use: Some IED attacks that would have been effective are made ineffective, and some IED attacks that would have been observed are never observed 14 Our instruments are justified based on the latter effect, suggesting that this effect is significant.

Suppose insurgents attempt exactly the same number of IED attacks no matter what countermeasures are used. However, jammer use also decreases the proportion of observed IEDs. Therefore, we will observe an apparent decrease in the number of IED attacks even

\footnotetext{
${ }^{14}$ It is likely that the effect on unobserved IED attacks dwarfs the effect on observed IED attacks becoming ineffective because the function of the jammer is to prevent the trigger from working at all, rather than to make the trigger work less effectively.
} 
though the number of IED attacks is actually constant. Reversing the logic, observing the same number of IED attacks necessarily means that more were attempted.

The $95 \%$ lower confidence bound on the estimate of $\Delta E_{t-1}$ is -0.235 : observed IED attacks decrease by $0.235 \%$ in response to a $1 \%$ decrease in IED effectiveness. This estimate is equivalent to $0.235 \%$ more IED attacks being made unobserved 15 As long as the effect of IED countermeasures on unobserved IED attacks is at least $23.5 \%$ of the effect of IED countermeasures on IED effectiveness - a reasonable assumption, because the purpose of IED countermeasures is to increase unobserved IED attacks rather than to decrease their effectiveness - the number of attempted IED attacks is actually increasing, but we do not fully observe the increase due to the increased proportion of unobserved IED attacks. ${ }^{16}$ Under this assumption, there is evidence that IED attacks may be a Giffen good.

This analysis, however, presumes that an unobserved IED attack costs an insurgent group the same amount of resources as an observed IED attack. An insurgent group may be able to recover the ordnance utilized in an unobserved attack, and may not subject itself to the same costs from the Coalition response because an unobserved attack is less risky to the insurgents than an observed one. Therefore, the costs of carrying out an attack that ends up unobserved may differ from one that is observed, not merely the benefits. Without more detailed data, it is impossible to determine if IED attacks are actually a Giffen good, or simply very close to being one. The statistical results indicate that IED attacks at least have inelastic demand and are inferior goods.

\subsection{Impact of IED Countermeasures}

Because demand for IED attacks is inelastic, a major way IED countermeasures are effective is in reducing non-IED attacks. The model finds that a decrease in effectiveness decreases

\footnotetext{
${ }^{15}$ We use the coefficient and standard error on $\Delta E_{t-1}$ because the lagged effect represents the assumption that the insurgents cannot react to countermeasures in the same time period.

${ }^{16}$ We would not necessarily expect to observe this effect contemporaneously because if the attack attempt is not observed, the insurgents may simply reuse the ordnance until they conduct an attack that does make contact with the Coalition. As noted above, the insurgents will not change their selection of the number of IED and non-IED attacks to conduct unless they realize this is happening in a systematic fashion. This effect also increases the percentage of non-radio control triggers in the observed population.
} 
non-IED attacks. In response to a Coalition negative effectiveness innovation, insurgents increase the number of IED attacks conducted (and because the proportion of unobserved IED attacks is higher, the number of observed IED attacks remains approximately the same - manifested by the coefficients in Equation 15 being insignificant). Therefore, countermeasures will appear to have no deterring effect, and in fact, both the number of effective attacks and the number of casualties due to IEDs will appear stable. However, analysis of these first-order effects ignore the additional resources required to inflict casualties.

Because additional resources are now required to inflict the same number of casualties, fewer resources are available, ceteris paribus, to carry out non-IED attacks. Therefore, the IED countermeasures have an effect in decreasing the number of casualties due to non-IED attacks. Because this effect is the primary mechanism by which IED countermeasures are effective, the elasticity of non-IED attacks to a decrease in IED effectiveness is high: 2.38 in the GMM model (over two months).

We use the constant term as a proxy for total insurgent resources. We find that, ceteris paribus the ability of insurgents to conduct IED attacks increases by $6 \%$ per month, and the ability of insurgents to conduct non-IED attacks increases by $13 \%$ per month. These increases may be due to increases in the amount of resources available to the insurgents, or technological progress that allows the insurgents greater efficiency in conducting these attacks.

In order to estimate the total effect IED countermeasures have had on non-IED attacks, we estimate the effect Coalition countermeasures have had, as measured through the instruments. An OLS regression of IED effectiveness on the instruments returns the coefficients determining $\Delta E_{t}$. Using these coefficients, it is possible to recover an estimate of the total effect IED countermeasures, as measured through decreases in IED effectiveness, have had on non-IED attacks. With the GMM estimate of an elasticity of 2.38 , we find that these countermeasures have prevented 1,997 non-IED attacks.

The first-order effect that these countermeasures have had (turning what would have been effective IED attacks into ineffective IED attacks) is estimated, using the above OLS 
regression of Equation 14, to be 1,504 IED attacks. These estimates show that the number of non-IED attacks prevented by IED countermeasures actually exceeds the number of IED attacks rendered ineffective by them. Furthermore, the countermeasures have no significant effect in reducing the number of IED attacks the insurgents attempt - they only reduce non-IED attacks in the data observed.

While these differences may seem small relative to the overall amount of money spent in the counter-IED fight, these estimates represent lower bounds on the effectiveness of IED countermeasures. We consider only jammers, a subset of the total counter-IED effort. There may be changes in Coalition techniques, tactics, and procedures, or innovations that reduce the expected number of casualties inflicted when casualties are taken. The actual impact of IED countermeasures on non-IED attacks is therefore likely to be considerably higher than the estimates presented here.

These estimates suggest that IED attacks are inferior and possibly even Giffen. This fact indicates that the proportion of IED attacks conducted decreases with an increase in resources. The insurgents face a complicated allocation problem - how to select the amount of ordnance to use against the Coalition now, and how much to hold in reserve for using in the power struggle that most expect would occur if the insurgents were to achieve their objectives and force the Coalition out of Iraq. The inferiority of IED attacks suggests that insurgents would prefer to be engaging in non-IED attacks, probably because the long-term gains from IED attacks are small 17 Non-IED attacks hold out the promise of control of territory, and with it, political legitimacy. We speculate it is for this reason that a systematic IED campaign is a tool of insurgent or terrorist organizations that are weak relative to the occupier, small in size, and politically extreme, rather than (for example) more mainstream organizations such as the larger-scale militias in Iraq that tend to conduct sectarian killings rather than IED attacks against Coalition forces.

\footnotetext{
${ }^{17}$ IED attacks do not directly help the insurgents, but instead they harm the Coalition. It seems likely that the biggest gain the insurgents receive is recruitment from publicizing their IED attacks.
} 


\section{Conclusion}

We have estimated the elasticities of IED and non-IED attacks in the war in Iraq to increased Coalition countermeasures, identified using IED triggers as instruments. We find that IED countermeasures have no significant impact in reducing the number of IED attacks conducted, but they do reduce the number of non-IED attacks the insurgency conducts. Theoretical considerations regarding the number of unobserved IED attacks suggest some limited evidence that IED attacks are a Giffen good. We find that a one percent decrease in IED effectiveness due to countermeasures decreases non-IED attacks by approximately two percent, and that IED countermeasures have thus prevented the insurgency from carrying out at least 1,997 non-IED attacks it otherwise would have. The number of non-IED attacks prevented exceeds the number of IED attacks rendered ineffective by the countermeasures, suggesting that the effectiveness of the $\$ 13$ billion spent on IED countermeasures has been significantly understated. 


\section{Appendix A: Data}

The data used are time series for the number of effective (defined as attacks that kill or wound at least one Coalition soldier) and ineffective IED attacks per month, the number of IEDs found and cleared, the total number of IED attacks per month, the number of total attacks per month (all methods), and the monthly percentages used of six IED triggers. The total number of IED attacks is defined to be the sum of ineffective and effective IED attacks plus the number of IEDs found and cleared. The number of non-IED attacks is the difference of the number of total attacks and the total number of IED attacks. Effectiveness is defined as the number of effective IED attacks divided by the number of total IED incidents (including IEDs found and cleared) 18 The sample is June 2004-April 2007.

The data on the total number of attacks per month are from Rebuilding Iraq: Integrated Strategic Plan Needed to Help Restore Iraq's Oil and Electricity Sectors (2007). The data on the number of effective, ineffective, and found/cleared IED incidents per month, as well as the time series for the six triggers observed, come from a Joint IED-Defeat Organization (JIEDDO) PowerPoint briefing ${ }^{19}$ In both cases, the data is collected from graphs in the documents. Although the data underlying these graphs is classified, the data on the graphs can be interpolated with better than $95 \%$ accuracy - therefore, measurement error above and beyond any errors that may exist in the underlying data is very limited 20 Although the scale bars on the graphs have been removed in the PowerPoint presentation and in the GAO report, the numbers can be inferred from reports which have stated several monthly numbers of IED attacks. Furthermore, the measurement error inherent in this process is considerably smaller than even the differences in the counts of IED attacks kept by different U.S. Department of Defense agencies. In this way, it is possible to create a highly accurate representation of trends in the IED fight from entirely unclassified, public data. ${ }^{21}$

\footnotetext{
${ }^{18}$ JIEDDO defines an effective attack as one that produces casualties.

${ }^{19}$ The briefing can be found on the Department of Defense website http://www.acq.osd.mil/ic/.

${ }^{20}$ Interpolation was conducted by counting the pixels on the graphs and generating an attacks per pixel value from several monthly values publicly announced by the Coalition.

${ }^{21}$ As a check on the quality of the data, the interpolated data agree closely with data presented in Atkinson (2007). Atkinson also identifies the six triggers: low-power radio control, high-power radio control, passive infrared, command wire, pressure plate, and cell phone.
} 


\begin{tabular}{|c|c|c|c|c|c|c|c|c|c|c|c|}
\hline Month & LPRC & HPRC & PI & CW & $\mathrm{PP}$ & $\mathrm{CP}$ & IED-FC & IED-I & IED-E & IED & NIED \\
\hline $6 / 04$ & 0.674 & 0.295 & 0.000 & 0.000 & 0.031 & 0.000 & 350 & 305 & 125 & 780 & 860 \\
\hline $7 / 04$ & 0.560 & 0.440 & 0.000 & 0.000 & 0.000 & 0.000 & 290 & 300 & 165 & 755 & 925 \\
\hline $8 / 04$ & 0.571 & 0.415 & 0.000 & 0.000 & 0.014 & 0.000 & 340 & 350 & 205 & 895 & 1985 \\
\hline $9 / 04$ & 0.477 & 0.490 & 0.000 & 0.000 & 0.033 & 0.000 & 350 & 395 & 190 & 935 & 1125 \\
\hline $10 / 04$ & 0.442 & 0.537 & 0.000 & 0.000 & 0.021 & 0.000 & 420 & 500 & 205 & 1125 & 1235 \\
\hline $11 / 04$ & 0.317 & 0.674 & 0.000 & 0.000 & 0.010 & 0.000 & 505 & 610 & 255 & 1370 & 1390 \\
\hline $12 / 04$ & 0.247 & 0.741 & 0.000 & 0.000 & 0.012 & 0.000 & 450 & 485 & 140 & 1075 & 865 \\
\hline $1 / 05$ & 0.429 & 0.548 & 0.000 & 0.000 & 0.023 & 0.000 & 620 & 625 & 175 & 1420 & 1360 \\
\hline $2 / 05$ & 0.160 & 0.830 & 0.000 & 0.000 & 0.010 & 0.000 & 515 & 545 & 160 & 1220 & 500 \\
\hline $3 / 05$ & 0.176 & 0.774 & 0.000 & 0.000 & 0.050 & 0.000 & 575 & 490 & 145 & 1210 & 330 \\
\hline $4 / 05$ & 0.180 & 0.788 & 0.000 & 0.000 & 0.033 & 0.000 & 570 & 615 & 210 & 1395 & 345 \\
\hline $5 / 05$ & 0.098 & 0.878 & 0.008 & 0.000 & 0.015 & 0.000 & 610 & 680 & 210 & 1500 & 600 \\
\hline $6 / 05$ & 0.069 & 0.873 & 0.029 & 0.000 & 0.029 & 0.000 & 550 & 615 & 190 & 1355 & 685 \\
\hline $7 / 05$ & 0.060 & 0.878 & 0.017 & 0.000 & 0.044 & 0.000 & 540 & 815 & 205 & 1560 & 780 \\
\hline $8 / 05$ & 0.050 & 0.849 & 0.006 & 0.000 & 0.095 & 0.000 & 595 & 850 & 210 & 1655 & 905 \\
\hline $9 / 05$ & 0.060 & 0.838 & 0.021 & 0.000 & 0.081 & 0.000 & 565 & 900 & 225 & 1690 & 790 \\
\hline $10 / 05$ & 0.019 & 0.755 & 0.044 & 0.000 & 0.181 & 0.000 & 785 & 990 & 245 & 2020 & 1000 \\
\hline $11 / 05$ & 0.014 & 0.736 & 0.025 & 0.000 & 0.222 & 0.004 & 790 & 730 & 140 & 1660 & 380 \\
\hline $12 / 05$ & 0.029 & 0.616 & 0.012 & 0.000 & 0.344 & 0.000 & 830 & 830 & 155 & 1815 & 665 \\
\hline $1 / 06$ & 0.025 & 0.635 & 0.017 & 0.147 & 0.141 & 0.035 & 780 & 645 & 140 & 1565 & 615 \\
\hline $2 / 06$ & 0.006 & 0.600 & 0.006 & 0.156 & 0.133 & 0.098 & 910 & 735 & 140 & 1785 & 595 \\
\hline $3 / 06$ & 0.021 & 0.573 & 0.006 & 0.154 & 0.156 & 0.089 & 1135 & 780 & 160 & 2075 & 805 \\
\hline $4 / 06$ & 0.019 & 0.488 & 0.037 & 0.085 & 0.224 & 0.147 & 1130 & 860 & 190 & 2180 & 1060 \\
\hline $5 / 06$ & 0.015 & 0.467 & 0.029 & 0.091 & 0.224 & 0.174 & 1215 & 1055 & 230 & 2500 & 1100 \\
\hline $6 / 06$ & 0.017 & 0.442 & 0.037 & 0.104 & 0.309 & 0.091 & 1155 & 1045 & 260 & 2460 & 1120 \\
\hline $7 / 06$ & 0.025 & 0.280 & 0.033 & 0.191 & 0.346 & 0.125 & 1230 & 1165 & 245 & 2640 & 1680 \\
\hline $8 / 06$ & 0.017 & 0.328 & 0.017 & 0.284 & 0.286 & 0.068 & 1275 & 1215 & 215 & 2705 & 1455 \\
\hline $9 / 06$ & 0.015 & 0.311 & 0.019 & 0.326 & 0.255 & 0.073 & 1365 & 1275 & 265 & 2905 & 2015 \\
\hline $10 / 06$ & 0.021 & 0.297 & 0.012 & 0.309 & 0.292 & 0.069 & 1375 & 1505 & 275 & 3155 & 2225 \\
\hline $11 / 06$ & 0.039 & 0.241 & 0.008 & 0.363 & 0.299 & 0.050 & 1395 & 1185 & 220 & 2800 & 1900 \\
\hline $12 / 06$ & 0.025 & 0.164 & 0.019 & 0.382 & 0.342 & 0.068 & 1305 & 1280 & 255 & 2840 & 2120 \\
\hline $1 / 07$ & 0.025 & 0.139 & 0.012 & 0.477 & 0.284 & 0.064 & 1235 & 1165 & 225 & 2625 & 2435 \\
\hline $2 / 07$ & 0.054 & 0.151 & 0.008 & 0.450 & 0.263 & 0.075 & 1465 & 1050 & 190 & 2705 & 1775 \\
\hline $3 / 07$ & 0.066 & 0.120 & 0.012 & 0.461 & 0.286 & 0.056 & 1645 & 1205 & 205 & 3055 & 1745 \\
\hline $4 / 07$ & 0.068 & 0.124 & 0.029 & 0.486 & 0.222 & 0.071 & 1415 & 1265 & 225 & 2905 & 1915 \\
\hline
\end{tabular}

Table 1: Data Used.

LPRC/HPRC: Low Power/High Power Radio Control

PI: Passive Infrared

CW: Command Wire

PP: Pressure Plate

CP: Cell Phone

IED-FC: IEDs Found/Cleared

IED-I: Ineffective IEDs

IED-E: Effective IEDs

IED: Total IED Attacks

NIED: Total Non-IED Attacks 


\section{Appendix B: Three-Stage Least Squares Estimation Results}

For Equation 12 , the coefficients on $\Delta E_{t}, \Delta E_{t-1}$ were found to be jointly insignificant. Although the coefficients on $\Delta N_{t-1}$ in both equations are jointly insignificant with $\Delta E_{t}, \Delta E_{t-1}$, we use $\alpha\left(\Delta I_{t-1}\right)+\beta\left(\Delta N_{t-1}\right)$, for some $\alpha$ and $\beta$, as one proxy for total resources, and we reject the null hypothesis that this proxy is insignificant in both equations. Therefore, we do not reduce the model further ${ }^{22}$ Setting the coefficients on $\Delta E_{t}, \Delta E_{t-1}$ equal to zero in the $\Delta I_{t}$ equation to improve efficiency produces the following results ${ }^{23}$

$$
\begin{gathered}
\widehat{\Delta I_{t}}=\underset{(0.019)}{0.051^{* *}}-\underset{(0.194)}{0.307} \Delta I_{t-1}-\underset{(0.063)}{0.060} \Delta N_{t-1} \\
T=34 \quad \bar{R}^{2}=0.155 \quad \hat{\sigma}=0.112 \\
\widehat{\Delta N_{t}}=\underset{(0.060)}{0.111^{*}}+\underset{(0.662)}{1.237^{*}} \widehat{\Delta E}_{t}+\underset{(0.362)}{0.651^{*}} \Delta E_{t-1}-\underset{(0.564)}{0.996^{*}} \Delta I_{t-1}-\underset{(0.186)}{0.157} \Delta N_{t-1} \\
T=34 \quad \bar{R}^{2}=0.243 \quad \hat{\sigma}=0.334 \\
\quad \text { (standard errors in parentheses) } \\
* * \text { significant at the } 5 \% \text { confidence level } \\
* \text { significant at the } 10 \% \text { confidence level }
\end{gathered}
$$

Although the 3SLS model does not show a high degree of statistical significance, adopting the $10 \%$ criterion for significance suggests qualitatively similar effects to those found in the GMM estimates. The parameter estimates are virtually identical, but the GMM level of confidence is much higher. This fact may be because GMM is more robust to nonstandard distributions and generally noisy data. These data do not fit normal economic relationships - there may be changes in regime, the dataset used is small, and the theoretical model requires a large number of variables to be correctly specified. All these factors suggest that the $10 \%$ level of confidence is justified - particularly when the $5 \%$ level of confidence is met by an alternative estimator that is similarly justified.

\footnotetext{
${ }^{22}$ Reducing the model further produces very similar results for the other coefficients, only increasing the magnitude of the coefficient on $\Delta I_{t-1}$.

${ }^{23}$ Diagnostic tests suggest that the residuals are normally distributed and not autocorrelated.
} 


\section{References}

Adamson, William G., "An Asymmetric Threat Invokes Strategic Leader Initiative: The Joint Improvised Explosive Device Defeat Organization," 2007. Industrial College of the Armed Forces Student Research Paper.

Atkinson, Rick, "Left of Boom: The Struggle to Defeat Roadside Bombs," The Washington Post, October 2007.

Battalio, Raymond C., John H. Kagel, and Carl A. Kogut, "Experimental Confirmation of the Existence of a Giffen Good," American Economic Review, Sep. 1991, 81 (4), 961-970.

Benmelech, Efraim and Claude Berrebi, "Attack Assignments in Terror Organizations and the Productivity of Suicide Bombers," 2007. NBER Working Paper No. W12910.

Bopp, Anthony E., "The Demand for Kerosene: A Modern Giffen Good," Economics Letters, Aug. 1983, 15 (4), 459-468.

Davis, Steven J., Kevin M. Murphy, and Robert H. Topel, "War in Iraq versus Containment," 2006. NBER Working Paper No. 12092.

Elliot, Graham, Thomas J. Rothenberg, and James H. Stock, "Efficient Tests for an Autoregressive Unit Root," Econometrica, July 1996, 64 (4), 813-36.

Ellis, Richard F., Richard D. Rodgers, and Bryan M. Cochran, "Joint Improvised Explosive Device Defeat Organization (JIEDDO): Tactical Successes Mired in Organizational Chaos; Roadblock in the Counter-IED Fight," March 2007. Joint Forces Staff College Student Paper.

Enders, Walter and Todd Sandler, "The Effectiveness of Antiterrorism Policies: A Vector-Autoregression-Intervention Analysis," American Political Science Review, December 1993, 87 (4), 829-844. 
and , The Political Economy of Terrorism, Cambridge: Cambridge University Press, 2006.

, and Jon Cauley, "Causality Between Transnational Terrorism and Tourism: The Case of Spain," Terrorism, January 1991, pp. 49-58.

Hansen, Lars Peter, "Large Sample Properties of Generalized Method of Moments Estimators," Econometrica, July 1982, 50, 1029-1054.

Hoy, Michael and Arthur J. Robson, "Insurance as a Giffen Good," Economics Letters, $1981,8(1), 47-51$.

Jaeger, David A. and M. Daniele Paserman, "Israel, the Palestinian Factions, and the Cycle of Violence," American Economic Review Papers and Proceedings, 2006, 96 (2), 45-49.

Jensen, Robert T. and Nolan H. Miller, "Giffen Behavior: Theory and Evidence," 2007. NBER Working Paper No. 13243.

Landes, William M., “An Economic Study of U.S. Aircraft Hijacking, 1961-1976," Journal of Law and Economics, 1978, 21, 1-31.

Leites, Nathan and Charles Wolf, Rebellion and Authority: An Analytic Essay on Insurgent Conflicts, Markham Publishing Company, 1970.

Rebuilding Iraq: Integrated Strategic Plan Needed to Help Restore Iraq's Oil and Electricity Sectors, Government Accountability Office, May 2007.

Rosendorff, B. Peter and Todd Sandler, "Too Much of a Good Thing? The Proactive Response Dilemma," Journal of Conflict Resolution, October 2004, 48 (5), 657-671.

Sandler, Todd and Walter Enders, "An Economic Perspective on Transnational Terrorism," European Journal of Political Economy, 2002.

Schelling, Thomas C., The Strategy of Conflict, Harvard University Press, 1960. 